\title{
The Use of (Double) Relaxation Oscillation SQUIDs as a Sensor
}

\author{
M.J. van Duuren, G.C.S. Brons, H.E. Kattouw, J. Flokstra and H. Rogalla \\ University of Twente, Dept. of Applied Physics, Low Temperature Div., P.O. box 217, 7500 AE Enschede, The Netherlands
}

\begin{abstract}
Relaxation Oscillation SQUIDs (ROSs) and Double Relaxation Oscillation SQUIDs (DROSs) are based on relaxation oscillations that are induced in hysteretic dc SQUIDs by an external L-R shunt. The relaxation frequency of a ROS varies with the applied flux $\Phi$, whereas the output of a DROS is a dc voltage, with a typical flux-to-voltage transfer of $\partial V / \partial \Phi \approx 1 \mathrm{mV} / \Phi_{0}$. The flux-to-frequency response of several ROSs has been measured and compared with theory for frequencies up to $7 \mathrm{GHz}$. Various DROS designs - a multi-loop direct coupling DROS, a DROS with a washer type signal SQUID and a DROS with gradiometric signal SQUID will be discussed in this paper. The integration of a DROS with a digital flux locked loop ("Smart DROS") will also be analyzed.
\end{abstract}

\section{INTRODUCTION}

Superconducting Quantum Interference Devices (SQUIDs) show a wide variety of applications, ranging from the detection of magnetic brain signals to the use as low noise pre-amps for various cryogenic detectors. Conventional dc SQUIDs are based on resistively shunted tunnel junctions and show a typical flux-tovoltage transfer of the order $\partial V / \partial \Phi=10-100 \mu V / \Phi_{0}$ and a typical flux noise level of $\sqrt{ } S_{\Phi}=1-10 \mu \Phi_{0} / \sqrt{H z}$. Due to the very low output voltage noise associated with this transfer $\left(\sqrt{ } S_{V}=\sqrt{ } S_{\Phi} \cdot \partial V / \partial \Phi \approx 0.1 \mathrm{nV} / \sqrt{\mathrm{Hz}}\right)$, direct voltage readout of a dc SQUID severely limits the system sensitivity because of the input voltage noise of the pre-amplifier. This explains the widespread use of ac flux and/or bias current modulation, cryogenic impedance matching circuitry and lock-in amplification. However, these modulation schemes pose several limitations, such as a limited bandwidth, crosstalk between adjacent channels and a complex system layout. Several approaches have been made in order to eliminate the need for modulation schemes [1]; this paper focuses on the category of relaxation oscillation driven dc SQUIDs.

The output signal of a Relaxation Oscillation SQUID (ROS) consists of voltage pulses with a frequency that depends on the applied flux. Its principle of operation and some experimental results are summarized in section II. A Double Relaxation Oscillation SQUID

Manuscript received August 27, 1996.

This work was supported by the Dutch Foundation for Scientific Research (NWO).
(DROS) converts a magnetic flux to a dc voltage with a much larger flux-to-voltage transfer than a conventional de SQUID, typically $\partial V / \partial \Phi=1 \mathrm{mV} / \Phi_{0}$. Thanks to this high transfer, direct voltage readout with a roomtemperature amplifier doesn't deteriorate the SQUID sensitivity. DROSs are the subject of section III.

A further step to facilitate SQUID readout is the integration and digitalization of the SQUID and its flux locked loop (FLL). Various single-chip digital SQUIDs have been presented in literature, e.g. [2] and [3], but these need an external clock signal for proper operation. A digital SQUID based on a DROS would not require such a clock, since the relaxation oscillations generated by the DROS itself can provide the clock pulses. A brief discussion of such a "Smart DROS" is given in Section IV. Finally, conclusions are given in section V.

\section{RELAXATION OSCILLATION SQUID}

\section{A. Principle of Operation}

A Relaxation Oscillation SQUID consists of a hysteretic dc SQUID that is shunted by an L-R circuit, as shown schematically in Fig. 1. A dc bias current $I_{b}$ causes the SQUID to oscillate between the superconducting and the resistive state if the ROS operation criterion

$$
\beta_{C}{ }^{*} \cdot\left(\frac{I_{b}}{I_{c}}\right)^{2}<1, \beta_{C}{ }^{*}=\frac{2 \pi \cdot I_{c} \cdot R_{s h}{ }^{2} \cdot C_{s q}}{\Phi_{0}},
$$

with $I_{c}$ the maximum critical current of the dc SQUID, $C_{s q}$ the SQUID capacitance and $\beta_{c}{ }^{*}$ the effective McCumber parameter for ROSs, is satisfied [4] , [5]. In Fig. 2, a numerical simulation of the relaxation

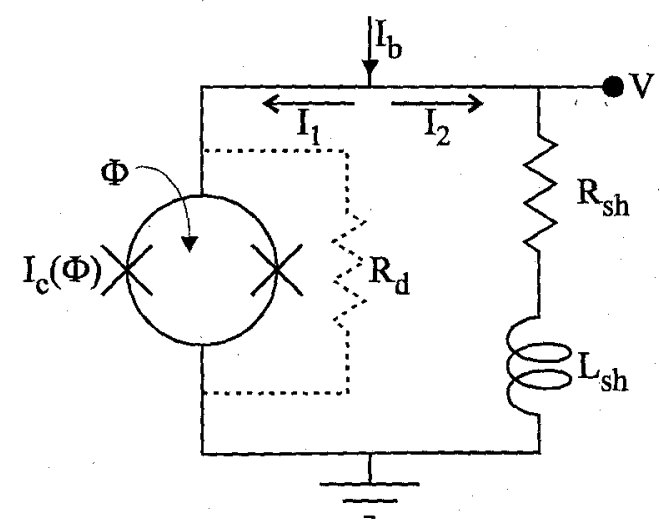

Fig. 1. Schematic diagram of a ROS. The resistor $R_{d}$ is optional. 


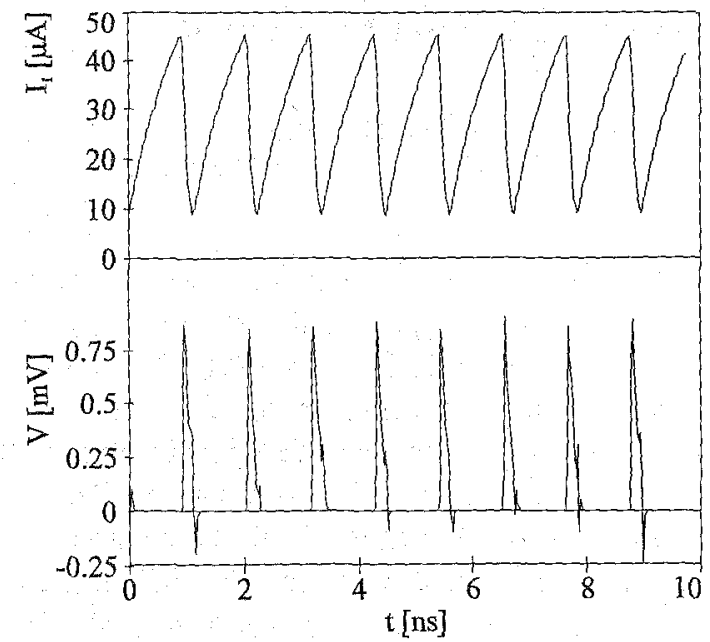

Fig. 2. Numerical simulation of relaxation oscillations in a ROS with $L_{s h}=2 \mathrm{nH}, \quad R_{s h}=2 \Omega, \quad C_{s q}=0.5 \mathrm{pF}, \quad I_{c}=45 \mu \mathrm{A}, \quad V_{g}=2.75 \mathrm{mV}$, $I_{b}=70 \mu \mathrm{A}$ and $R_{d}=25 \Omega$. The de SQUTD was modeled as a single junction.

oscillation process is shown. In this simulation, an additional damping resistor $R_{d}$ was added to the ROS in order to damp parasitic resonances between $L_{s h}$ and $C_{s q}$, since these resonances can degrade the ROS operation considerably [4].

The frequency of the relaxation oscillations, $f_{R O}$, depends on the bias current $I_{b}$, on the ROS time constant $L_{s h} / R_{s h}$, and on the critical current $I_{c}(\Phi)$ of the SQUID, and can be approximated by [6]

$$
f_{R O} \approx \frac{R_{s h}}{L_{s q}} \cdot \frac{1}{\ln \left[I_{b} /\left(I_{b}-I_{c}(\Phi)\right)\right]} .
$$

Since $I_{c}(\Phi)$, the critical current of the dc SQUID, depends on the applied flux $\Phi$, the output frequency of a ROS depends on $\Phi$.

The dominant noise source in a properly designed ROS is the thermal noise on the critical current. Its contribution decreases for increasing relaxation frequencies, at relaxation frequencies in the order of $1 \mathrm{GHz}$, the theoretical sensitivity of a ROS is comparable to that of standard dc SQUTDs [6]. A more comprehensive description of the theoretic background of the ROS can be found in [4].
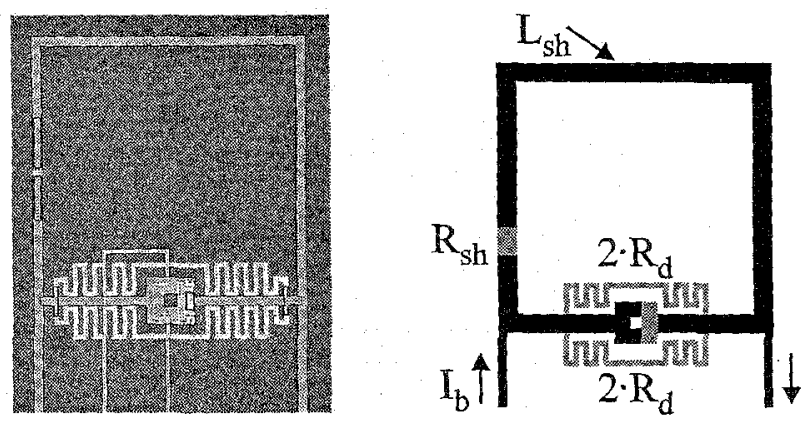

Fig. 3. Micrograph and scheme of a $1 \mathrm{GHz}$ ROS.

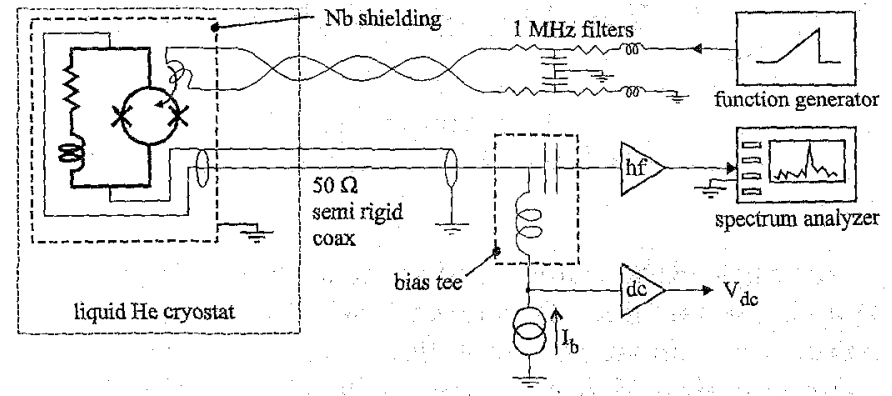

Fig. 4. Measurement setup for $f_{R O}(\Phi)$ characterization of ROSs.

\section{B. Experimental ROS Results}

Various ROSs with nominal relaxation frequencies of 1 and $10 \mathrm{GHz}$ (i.e. $L_{s h} / R_{s h}=1$ or $0.1 \mathrm{~ns}$ ) and different damping resistors $R_{d}$ have been designed, fabricated and characterized [4]. In Fig. 3, a micrograph of a $1 \mathrm{GHz}$ ROS is shown and Fig. 4 depicts the experimental setup that was used to characterize the ROSs. By the use of a bias tee, one coaxial line could be used for both the readout of the high frequency output signal of the ROS and for applying the bias current.

The experimental $f_{R O}(\Phi)$ characteristics of all ROSs have been fitted with theory according to a model that is presented and discussed in [4]; in fact it is a modified version of eq. (2). A typical example of such a fit for a $1 \mathrm{GHz}$ ROS is shown in Fig. 5. Also the measurements with the $10 \mathrm{GHz}$ ROSs show a good agreement with theory, even for the highest observed relaxation frequencies of about $7 \mathrm{GHz}$.

A FLL based on FM demodulation of the ROS output signal has also been realized [4]. The sensitivity, $\sqrt{S_{\Phi}} \approx 2.5 \mu \Phi_{0} / \sqrt{\mathrm{Hz}}$ (corresponding to an energy resolution of $\varepsilon=S_{\Phi} / 2 L_{s q} \approx 600 h$ ) for a $1 \mathrm{GHz}$ ROS and $\sqrt{S_{\Phi}} \approx 4 \mu \Phi_{0} / \sqrt{\mathrm{Hz}}$ for a $10 \mathrm{GHz}$ ROS, was limited by the noise of the FM demodulator.

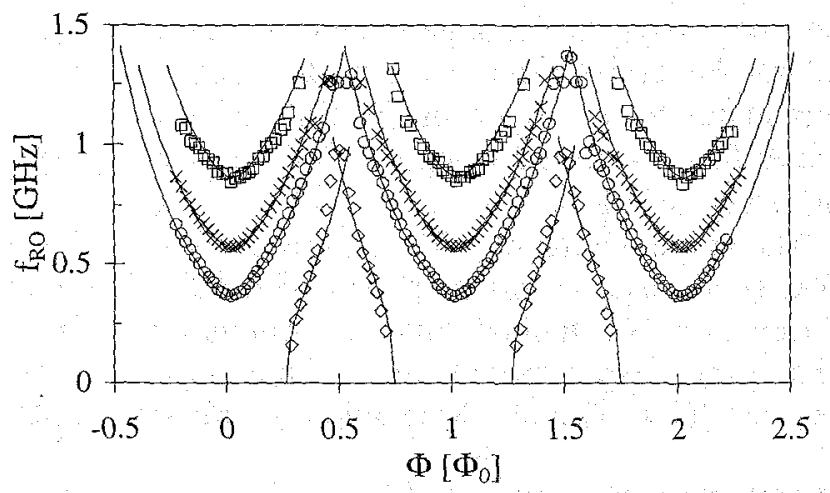

Fig. 5. Experimental (markers) and theoretical (lines) $f_{R O}(\Phi)$ characteristics of a $1 \mathrm{GHz}$ ROS for different values of the bias current. 


\section{DOUBLE RELAXATION OSCILLATION SQUID}

\section{A. Principle of Operation}

In a DROS, the $L_{s h}-R_{s h}$ circuit shunts two SQUIDs in series, as shown in Fig. 6 . The signal flux $\Phi$ is applied to only one of the two SQUIDs, the signal SQUID. The critical current of the other SQUID, the reference SQUID, is trimmed by means of an adjustable flux $\Phi_{\text {ref. }}$.

If the DROS is dc biased, relaxation oscillations can occur under the same condition and with the same frequency as in the case of a ROS. These oscillations cause the SQUID with the smallest critical current to switch to the voltage state periodically, the other SQUID remains superconducting. So, if $I_{c, s i g n}(\Phi)<I_{c, \text { ref }}$, the signal SQUID oscillates while the reference SQUID stays in the zero-voltage state; the DROS output is $V=0$ in this case. Oppositely, when $\Phi$ is changed so that $I_{c, s i g n}(\Phi)>I_{c, \text { ref }}$, the reference SQUID oscillates, causing a time-averaged voltage $V_{c}$ at the output.

The width of the transition from $V=0$ to $V=V_{c}$, and thus the flux-to-voltage transfer $\partial V / \partial \Phi$, is determined by the thermal spread in the critical currents of both SQUIDs [5], [6]. In practice, $\partial V / \partial \Phi$ is in the order of $1 \mathrm{mV} / \Phi_{0}$, as illustrated in the experimental $V(\Phi)$ characteristic of Fig. 7. This large flux-to-voltage transfer enables FLL operation based on direct voltage readout, without flux modulation. As in the case of a ROS, the relaxation frequency of a DROS should be around $1 \mathrm{GHz}$ for optimum sensitivity [5], [6].

\section{B. Magnetometers and Gradiometers based on DROSs}

For utilization with external flux transformers, DROSs with a washer type signal SQUDD and integrated 25-turn input coil have been made. A micrograph and the scheme of this DROS layout are shown in Fig. 8. An external $R_{i n} C_{\text {in }}$ shunt $\left(R_{i n}=150 \Omega\right.$ and $C_{i n}=1 \mathrm{nF}$, both $\mathrm{SMD}$ ) across the input coil improved the DROS sensitivity considerably, probably

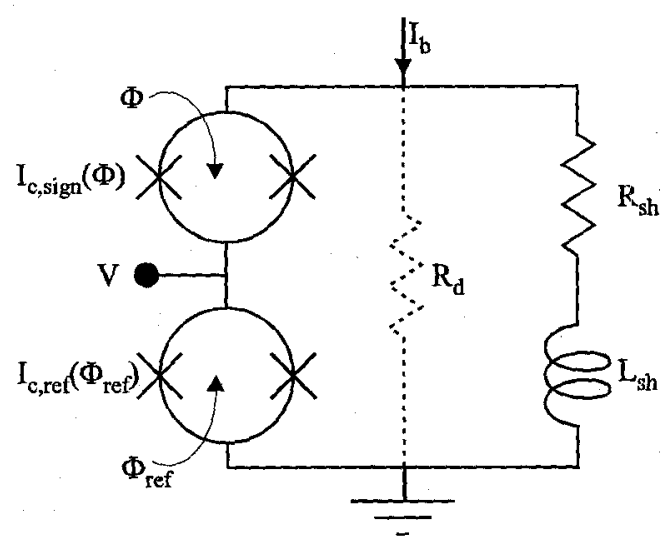

Fig. 6. Schematic diagram of a DROS.

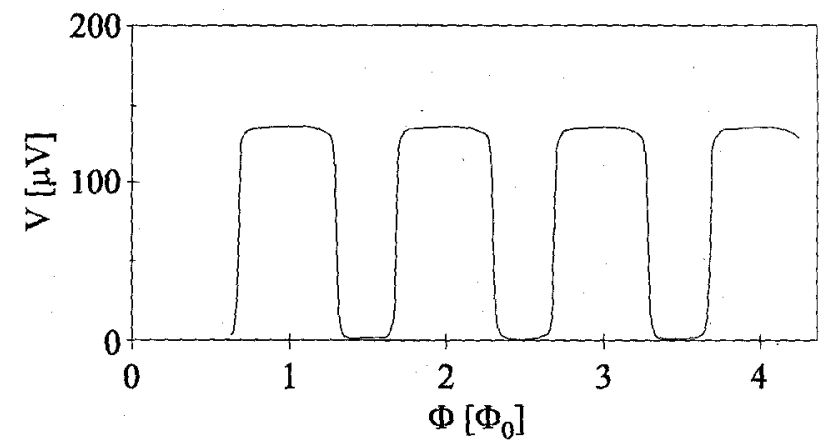

Fig. 7. Experimental $V(\Phi)$ characteristic of a DROS.

by damping of resonances in the input coil [7]. Operated in a FLL with direct voltage readout, the DROSs showed a white flux noise level of $S_{\Phi} \approx 4.5 \mu \Phi_{0} / \sqrt{\mathrm{Hz}}$ typically. Combined with the SQUID inductance of $L_{s q}=240 \mathrm{pH}$, this corresponds to an energy resolution $\varepsilon=275 h$. Equipped with an axial wire-wound first-order gradiometer with a diameter of $2 \mathrm{~cm}$ and a baseline of $4 \mathrm{~cm}$, these DROSs were used in a three-channel DROS gradiometer system [8], [9]. The measured field sensitivity, $\sqrt{ } S_{B}=4 \mathrm{fT} / \sqrt{\mathrm{Hz}}$, was not influenced by possible interference of the relaxation oscillations in adjacent channels, which demonstrates the usability of DROSs in multi-channel systems.

In this three-channel gradiometer system, the three DROS chips were shielded by individual $\mathrm{Nb}$ modules to minimize parasitic pickup of magnetic fields. Recently, we developed DROSs with a gradiometric signal SQUID and integrated $2 \times 25$ turns input coil. The gradiometric layout of the signal SQUID and a superconducting shielding loop around the reference SQUID greatly reduce the response to homogeneous magnetic fields. Thanks to this field immunity, this DROS can be operated without extensive shielding of the chip. This was illustrated by mounting a gradiometric DROS directly on the glass substrate of a planar gradiometer with pickup loops of $2 \mathrm{~cm} \times 2 \mathrm{~cm}$. Inside a magnetically shielded room, the field of a
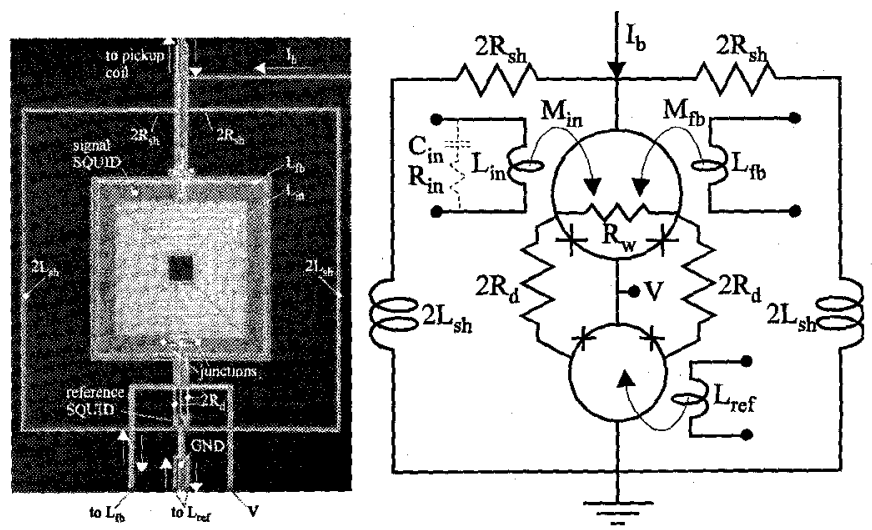

Fig. 8. Micrograph and scheme of DROS with 25-turn input coil. 


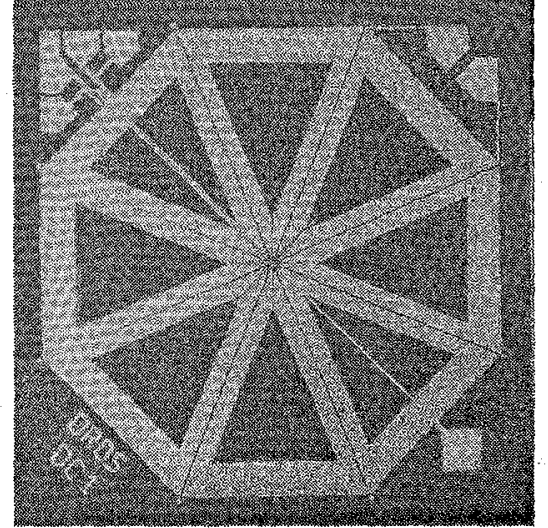

Fig. 9. Micrograph of a multi-loop direct coupling DROS. The actual size is $9 \mathrm{~mm} \times 9 \mathrm{~mm}$.

human heart could be detected very clearly with this configuration. Detailed performance data will be published elsewhere.

A third DROS layout that has been under study is the multi-loop direct coupling DROS that is shown in Fig. 9. In this DROS design, the signal SQUID combines a large effective area, $A_{\text {eff }}=4.1 \mathrm{~mm}^{2}$, with a relatively small SQUID inductance of $L_{s q} \approx 400 \mathrm{pH}$, since the SQUID inductance is composed of eight large loops connected in parallel [10]. Thanks to the large effective area, this DROS magnetometer can be used without external flux transformer. The measured magnetic field sensitivity of this device was $\sqrt{ } S_{B}=7 \mathrm{fT} / \sqrt{\mathrm{Hz}}$ in FLL. The direct coupling DROS is discussed in more detail in [11].

\section{IV. "SMART DROS"}

The pulsed nature of the DROS output is well suited for digital processing, which offers the possibility of a digital SQUID with integrated on-chip FLL. A potential arrangement, in which a DROS drives a digital updown counter, is sketched in Fig. 10. The voltage pulses caused by the signal SQUID drive the UP gate of the counter, whereas the voltage pulses across the reference SQUID cause the counter to count down one unit. The output of the counter provides the feedback flux $\Phi_{f b}$ that is added to the signal flux $\Phi$. In operation, the system will be flux locked at the dynamic equilibrium were the switching probability of both SQUDs is equal.

Such a digital DROS would have the same advantages as the digital SQUIDs that were presented in [2] and [3], without requiring an external clock. Therefore, the operation frequency can be much higher than in those systems, which increases the sensitivity and improves the dynamic behavior of the system. Numerical simulations of a DROS with an SFQ counter of the type that was presented in [3] are in progress.

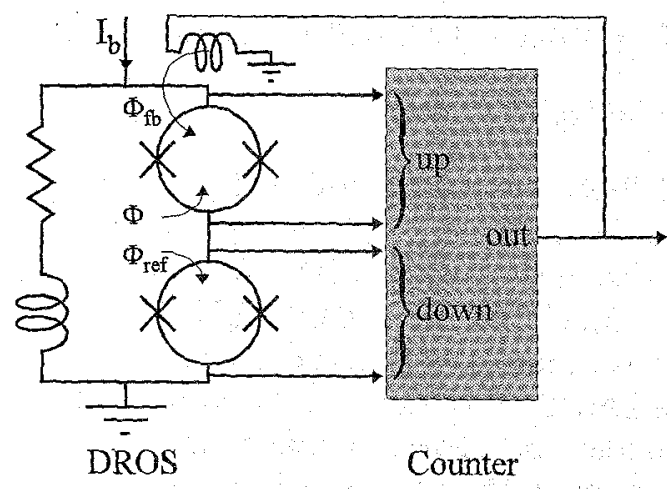

Fig. 10. Scheme of a "Smart DROS".

\section{CONCLUSIONS}

A ROS converts a magnetic flux to a frequency. For optimum sensitivity, the relaxation oscillation frequency should be in the $\mathrm{GHz}$ range. Experimental frequency-flux characteristics can be fitted very well with theory, even for frequencies as high as $7 \mathrm{GHz}$. However, the readout of the high frequency output of a ROS can be difficult in practice, in contrast to the direct voltage readout scheme of a DROS. This direct voltage readout is made possible by the high flux-to-voltage transfer of DROSs. Several DROS designs have been tested successfully in practice. Moreover, DROSs offer the possibility of on-chip integration with a digital FLL. Such a "Smart DROS" would not need any external clock signals for operation.

\section{REFERENCES}

[1] D. Drung, Supercond. Sci. Technol, vol. 4, pp. 377-385, 1991.

[2] N. Fujimaki; H. Tamura, T. Imamura, and S. Hasuo, IEEE Trans. Electron Devices, vol. 35, pp. 2412-2418, 1988.

[3] M. Radparvar, IEEE Trans. Appl. Superconduct., vol. 4, pp. 87-91, 1994.

[4] M.J. van Duuren, D.J. Adelerhof, G.C.S. Brons, J. Kawai, G. Uehara, H. Kado, J. Flokstra, and H. Rogalla, J. Appl. Phys., in press.

[5] D.J. Adelerhof, J Kawai, G. Uehara, and H. Kado, Rev. Sci Instrum., vol. 66, pp. 2631-2637, 1995.

[6] D.J. Adelethof, H. Nijstad, J. Flokstra, and H. Rogalla, J. Appl. Phys., vol. 76, pp. 3875-3886, 1994.

[7] K. Enpuku and K. Yoshida, J. Appl. Phys., vol. 69, pp. 7295$7300,1991$.

[8] M.J. van Duuren, Y.H. Lee, D.J. Adelerhof, J. Kawai, H. Kado, J. Flokstra, and H. Rogalla, IEEE Trans. Appl. Superconduct., vol. 6, pp. 38-44, 1996.

[9] M.J. van Duuren, D.J. Adelerhơf, Y.H. Lee, J. Flokstra, and H. Rogalla, Proc. EUCAS '95 Conference, Edinburgh, UK, pp. 1507-1510, 1995.

[10] D. Drung, S. Knappe, and H. Koch, J. Appl. Phys., vol. 77, pp. 4088-4098, 1995.

[11] D.J. Adelerhof, H. Wichers, C. Brons, M. van Duuren, D Veldhuis, J. Flokstra, and H. Rogalla, Proc. ISEC '95 Conference, Nagoya, Japan, pp. 371-373, 1995. 